\title{
NONLINEAR OPEN LOOP OPTIMAL TRACKING USING MAGNETOSTRICTIVE TRANSDUCERS
}

\author{
William S. Oates* \\ Center for Research in Scientific Computation \\ Department of Mathematics \\ North Carolina State University \\ Raleigh, North Carolina 27695 \\ Email:wsoates@ncsu.edu
}

\author{
Ralph C. Smith \\ Center for Research in Scientific Computation \\ Department of Mathematics \\ North Carolina State University \\ Raleigh, North Carolina 27695 \\ Email: rsmith@ncsu.edu
}

\begin{abstract}
The design of high performance smart material devices requires control laws that can achieve stringent performance criteria required in many industrial, automotive, aerospace and biomedical applications. Moderate to high field inputs are often required which introduces nonlinear, hysteretic constitutive behavior. This often cannot be effectively compensated using linear control theory. In the present work, a nonlinear optimal control methodology is developed for tracking a reference trajectory when nonlinear, hysteretic constitutive behavior is significant. For brevity, we focus on open loop control for regimes when disturbances are minimal. The nonlinear control design is investigated on magnetostrictive rod-type actuators by implementing a homogenized energy model. Significant emphasis is place on material behavior at multiple length scales to produce an accurate and efficient model to compensate for nonlinear, hysteretic magnetostrictive constitutive behavior.
\end{abstract}

\section{INTRODUCTION}

Smart or active materials exhibit deformations when exposed to electrical, magnetic, or thermal fields. The field-coupled material behavior offers several advantages over conventional actuating devices that are often large, heavy and complex. For example, piezoelectric stack actuators offer broadband capability, compactness and high forces which have been implemented in morphing helicopter rotor systems for reductions in vibration

*Address all correspondence to this author. and improved aerodynamic performance [1]. In industrial applications, magnetostrictive materials such as Terfenol-D are often used for precision machining operations [2,3]. Shape memory alloys (SMAs) have been investigated for use as microactuators for minimally invasive surgical procedures. A good review of biomedical devices that exploit SMAs is given by Duerig [4].

Although significant advances have been made in developing smart material devices, several challenges remain. For example, achieving a desired performance specification requires developing a control strategy that accurately accounts for fieldcoupled material behavior. Many active materials exhibit nonlinearities and hysteresis at field levels required to compete with conventional actuators. If this behavior is neglected, instabilities may result from a phase lag between the input field and actuator displacement. By introducing nonlinear, hysteretic constitutive behavior directly into the control design, performance is improved by minimizing the amount of feedback required to compensate for unmodeled dynamics.

Model-based control designs for hysteretic systems are not new and have received considerable attention in areas relative to adaptive, classical and optimal control [5-8]. These models typically address hysteretic material behavior by implementing Preisach operators [8] or domain wall models [9]. Although Preisach models can reasonably predict nonlinear, hysteretic material behavior observed in smart materials such as piezoelectric, magnetostrictive and shape memory alloys, a significant number of non-physical parameters are necessary to model minor loop hysteresis. Domain wall models avoid this issue by incorporating
Copyright (c) 2005 by ASME 
energetics at the domain length scale to predict macroscopic material behavior, but extensive modifications are required to guarantee closure of minor loops.

In the present work, a model-based optimal control design is developed using a homogenized energy model which overcomes several of the above deficiencies. The model and control design are sufficiently general to encompass a wide range of smart material actuators. For purposes of demonstrating the control design, we focus here on magnetostrictive transducers used in high speed machining operations. The constitutive model is briefly summarized first. A structural model is then developed to couple the magnetostrictive transducer to a damped oscillator used to represent the structure or device being actuated (in this case, the part being machined). The control theory is then developed. Tracking performance is demonstrated using linear optimal control theory and compared to the nonlinear optimal control approach. Significant improvements in precision position control are obtained when the magnetostrictive constitutive behavior is compensated properly.

\section{Magnetostrictive Homogenized Energy Model}

The macroscopic constitutive behavior is predicted by implementing a homogenized energy framework. The focus here is on uniaxial loading of rod-type actuators and thus the fields and material coefficients have been reduced to scalar coefficients or distributed variables in the direction of loading. The theory focuses on energy relations at the mesoscopic or lattice length scale. A stochastic homogenization technique is then implemented to determine macroscopic thermodynamic parameters from a distribution of local material properties. The local field behavior is given by

$$
\bar{M}=\chi_{m}\left(H+H_{I}\right)+\delta M_{R}
$$

where the local magnetization is $\bar{M}$, the applied magnetic field is defined by $H$, and $\chi_{M}$ is the magnetic susceptibility. The variable $M_{R}$ denotes the local magnetization variant and $\delta=1$ for local variants aligned in the positive direction and $\delta=-1$ for local variants aligned in the negative direction. The variable $H_{I}$ represents local variations in the applied field due to material inhomogeneities.

The macroscopic field quantities are determined by assuming a distribution of local fields properties given in Eq. (1). Variations in local properties may originate from a number of sources including mismatch in grain orientation, defects along grain boundaries, twinned domain structures, and inhomogeneities in composition. To model these multi-scale phenomena efficiently, the relation

$$
[M(H)](t)=\int_{0}^{\infty} \int_{-\infty}^{\infty} v\left(H_{c}, H_{I}\right)\left[\bar{M}\left(H+H_{I} ; H_{c}, \xi\right)\right](t) d H_{I} d H_{c}
$$

is employed to obtain macroscopic field behavior where $H_{c}$ represents the coercive field, $\xi$ represents the initial distribution of the local variants, and $v\left(H_{c}, H_{I}\right)$ denotes the distribution of coercive and interaction fields. To reduce parameter estimation, the following form of the distribution is used,

$$
\mathrm{v}\left(H_{c}, H_{I}\right)=c_{1} e^{-\left[\ln \left(H_{c} / \bar{H}_{c}\right) / 2 c\right]^{2}} e^{-H_{I}^{2} / 2 b^{2}}
$$

where $\bar{H}_{c}$ is the average coercive field, $c$ quantifies the coercive field variability, $b$ is the variance of the interaction field, and $c_{1}$ is a scaling parameter. Model comparison to experimental results can be found in [10].

The constitutive law describing the uniaxial stress field is

$$
\sigma=Y^{M} \varepsilon-a_{1}\left(M-M_{0}\right)^{2}
$$

where $Y^{M}$ is the elastic modulus at constant magnetization, $\varepsilon$ is the linear strain component, $a_{1}$ is the magnetostrictive coefficient. Again, $M$ is the total magnetization while $M_{0}$ includes effects from a permanent magnet and the initial magnetized state of the material.

The stress computed using Eq. (4) includes linear stressstrain behavior as well as nonlinear and hysteretic dependence on the magnetic field. It does not incorporate internal damping or spatial dependence. This is incorporated in the following section.

\section{Structural Model}

To facilitate the control design, the constitutive relations given by Eqs. (2) and (4) are used to develop a system model that quantifies forces and displacements when a magnetic field is applied to the magnetostrictive transducer. The PDE model is first presented and then formulated as an ODE by the use of a finite element discretization. A damped oscillator serves as boundary conditions at the end of the actuator to represent the structure being machined. The structural model is illustrated in Fig. 1 .

A balance of forces for the structural model is given by the relation [11]

$$
\rho A \frac{\partial^{2} w}{\partial t^{2}}=\frac{\partial N_{t o t}}{\partial x}
$$




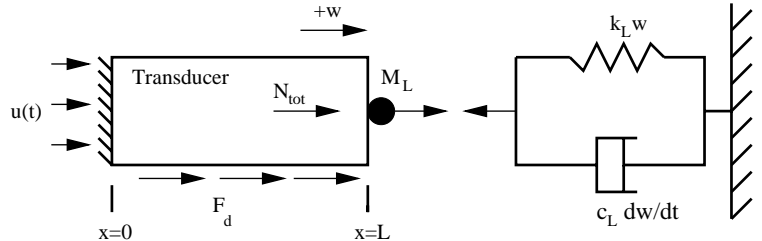

Figure 1. MAGNETOSTRICTIVE TRANSDUCER WITH DAMPED OSCILLATOR USED TO QUANTIFY LOADS DURING THE MACHINING OPERATION. DISTURBANCE FORCES ALONG THE ACTUATOR ARE GIVEN BY $F_{d}$ AND THE CONTROL INPUT IS $u(t)$.

where the density of the actuator is given by $\rho$, the cross-section area is $A$ and the displacement is denoted by $w$. The total force $N_{\text {tot }}$ acting on the actuator is given by the relation

$$
N_{t o t}(t, x)=Y^{M} A \frac{\partial w}{\partial x}+c_{D} A \frac{\partial^{2} w}{\partial x \partial t}+F_{m a g}+F_{d}
$$

where the elastic restoring force is given by the first term on the right hand side of the equation and a Kelvin-Voigt damping force is given by the second term. The linear elastic strain component is defined by $\varepsilon=\frac{\partial w}{\partial x}$. The external disturbance force is given by $F_{d}$. The coupling force $F_{\text {mag }}$ represents forces generated by applied magnetic field where

$$
F_{m a g}=A a_{1}\left(M-M_{0}\right)^{2} \text {. }
$$

Although the modeling framework includes disturbance loads, loads in excess of the ferromagnetic coercive stress were necessary to induce noticeable errors in the tracking performance for the system under consideration. Therefore, the disturbance loads were set to zero in the simulations.

Following Fig. (1), the boundary conditions are defined by a zero displacement at $x=0$ and the balance of forces at $x=L$ gives

$$
N_{t o t}(t, L)=-k_{L} w(t, L)-c_{L} \frac{\partial w}{\partial t}(t, L)-M_{L} \frac{\partial^{2} w}{\partial x \partial t}(t, L) .
$$

The initial conditions are $w(0, x)=0$ and $\frac{\partial w}{\partial x}(0, x)=0$.

The PDE model given by Eq. (5) can be written in weak form and spatially discretized using finite elements to obtain an ODE system of equations. Details of the technique are given $[10,11]$. This yields the following set of equations in matrix form

$$
\mathbb{M} \ddot{\mathbf{w}}+\mathbb{C} \dot{\mathbf{w}}+\mathbb{K} \mathbf{w}=F_{c} \mathbf{b}+\mathbf{f}_{\mathbf{d}}
$$

where the matrices $\mathbb{M} \in \mathbb{R}^{N \times N}, \mathbb{C} \in \mathbb{R}^{N \times N}$ and $\mathbb{K} \in \mathbb{R}^{N \times N}$ denote the mass, damping and stiffness matrices, respectively. The nodal displacements are denoted by $\mathbf{w} \in \mathbb{R}^{N}$ and the vectors $\mathbf{b}, \mathbf{f}_{\mathbf{d}} \in \mathbb{R}^{N}$ includes the basis functions related to the control input and disturbance loads, respectively. The number of finite elements is defined by $N$.

Equation (9) can be written in terms of a set of first order equations for developing the control law. This results in

$$
\dot{x}(t)=A x(t)+[B(u)](t)+G(t)
$$

where $x(t)=[\dot{\mathbf{w}}, \mathbf{w}]^{T}$ should not to be confused with the coordinate $x$. The matrix $A$ includes the mass, damping and stiffness properties of the system given in Eq. (9) and $[B(u)](t)$ includes the nonlinear input where $u(t)$ is defined as the magnetic field. External disturbances are given by $G(t)$. Equation (10) is used in the following sections to develop the control law.

\section{Control Design}

First the general tracking problem is briefly summarized to provide relations used in developing the nonlinear optimal tracking control law. The output to Eq. (10) is a linear combination of the states,

$$
y(t)=C x(t)
$$

where it is assumed that only displacement at the end of the transducer can be measured. The cost functional

$$
\begin{aligned}
\bar{J}= & \frac{1}{2}\left(C x\left(t_{f}\right)-r\left(t_{f}\right)\right)^{T} P\left(C x\left(t_{f}\right)-r\left(t_{f}\right)\right) \\
& +\int_{t_{0}}^{t_{f}}\left[H-\lambda^{T}(t) \dot{x}(t)\right] d t
\end{aligned}
$$

is defined to penalize the measurable state and the control input where $P$ penalizes large terminal values on the observable state, $H$ is the Hamiltonian, $r(t)$ is the reference signal, and $\lambda(t)$ is a set of Lagrange multipliers. The Hamiltonian is 


$$
\begin{aligned}
H= & \frac{1}{2}\left[(C x(t)-r(t))^{T} Q(C x(t)-r(t)) u^{T}(t) R u(t)\right] \\
& +\lambda^{T}[A x(t)+[B(u)](t)+G(t)]
\end{aligned}
$$

where penalities on the observable state and inputs are given through the variables $Q$ and $R$, respectively.

To determine the optimal input, the minimum of Eq. (12) must be determined under the constraint of Eq. (10). An unconstrained optimization problem is developed by introducing the costate system $[12,13]$

$$
\dot{\lambda}(t)=-A^{T} \lambda(t)-C^{T} Q C x(t)+C^{T} Q r(t) .
$$

The optimal input control is determined from the stationary condition on the Hamiltonian which results in

$$
u(t)=-R^{-1}\left(\frac{\partial B(u)}{\partial u}\right)^{T} \lambda(t)
$$

The boundary conditions are applied to the state at the initial time and the final time on the costate

$$
\begin{aligned}
& x\left(t_{0}\right)=x_{o} \\
& \lambda\left(t_{f}\right)=C^{T} P\left(C x\left(t_{f}\right)-r\left(t_{f}\right)\right) .
\end{aligned}
$$

These equations result in a two-point boundary value problem that are in general challenging to solve for large $N$. In the following section, linear control theory is implemented by solving the suboptimal infinite horizon problem which is often done to simplify the solution technique. Linear control theory is shown to provide poor control authority when nonlinear hysteretic material behavior is present. The two-point boundary value problem is then directly addressed in the nonlinear optimal tracking control method. By introducing the constitutive behavior directly into the optimality system, it is shown that the control input effectively compensates for nonlinearity and hysteresis.

\section{Linear Tracking Control}

The linear control design requires simplifying the nonlinear input given by the coupling force in Eq. (7). These terms are linearized by taking a Taylor series expansion about the fully magnetized state of the material. The control input in this case is limited to a small time varying input, thus only first order variations are included in the control law. In the case of magnetostrictive materials, linearization is taken about $M_{0}$.

The linearized forces are

$$
F_{m a g} \simeq 2 A a_{1} M_{0} \Delta M
$$

where $\Delta M=M-M_{0}$.

A linear magnetic constitutive law is introduced such that the control input is given by an applied magnetic field

$$
\Delta M=M-M_{0}=\chi_{m} \Delta H
$$

The change in magnetic field is denoted by $\Delta H$ where $\chi_{m}$ is the magnetic susceptibility. This yields

$$
F_{m a g} \simeq 2 A a_{1} M_{0} \chi_{m} \Delta H .
$$

With this approximation, the corresponding first-order system is

$$
\begin{aligned}
& \dot{y}(t)=A y(t)+B u(t)+G(t) \\
& y(0)=y_{0} .
\end{aligned}
$$

The state constraint in Eq. (20) and the linear version of the adjoint condition in Eq. (14) yields the optimality system

$$
\begin{aligned}
& {\left[\begin{array}{l}
\dot{y}(t) \\
\dot{\lambda}(t)
\end{array}\right]=\left[\begin{array}{cc}
A & -B R^{-1} B^{T} \\
-C^{T} Q C & -A^{T}
\end{array}\right]\left[\begin{array}{l}
y(t) \\
\lambda(t)
\end{array}\right]+\left[\begin{array}{l}
G(t) \\
C^{T} Q r(t)
\end{array}\right]} \\
& y\left(t_{0}\right)=y_{0} \\
& \lambda\left(t_{f}\right)=\Pi_{f} y\left(t_{f}\right) .
\end{aligned}
$$

For periodic, steady-state behavior, we consider the performance index

$$
J(u)=\int_{0}^{\tau} \frac{1}{2}\left[y^{T}(t) Q y(t)+u^{T}(t) R u(t)\right] d t
$$

where $\tau$ is the largest commensurate frequency. 
Since the input operator $B$ is linear, a fundamental solution matrix is determined by solving the algebraic Ricatti equation

$$
A^{T} \Pi+\Pi A-\Pi B R^{-1} B^{T} \Pi+Q=0 .
$$

The optimal control input is then defined by

$$
u^{*}(t)=-R^{-1} B^{T}[\Pi y(t)-v(t)]
$$

where the variable $\mathrm{v}(t) \in \mathbb{R}^{2 N}$ is the solution to the auxiliary differential equation

$$
\begin{aligned}
& \dot{v}(t)=-\left[A-B R^{-1} B^{T} \Pi\right]^{T} v(t)+\Pi G(t) \\
& \vee\left(t_{f}\right)=0 .
\end{aligned}
$$

In the finite time problem, the condition on the final time is substituted for an initial condition given by $v(0)=v(\tau)$. This approach requires restricting the disturbance force to be periodic where $\tau$ denotes the fundamental period for all frequencies present. Details regarding this approach are given in $[14,15]$.

The suboptimal control trajectory determined by Eq. (24) is used in the following numerical examples to illustrate the need for nonlinear control when the input field reaches a magnitude that induces hysteresis.

Numerical Results: Linear Model Tracking control is demonstrated using the linear control theory to illustrate performance in tracking a reference when small or moderate to large fields are applied. The Terfenol rod is fully magnetized in the constitutive model prior to control simulation. The penalties on the state and input are given by $Q=1 \times 10^{12}$ and $R=1 \times 10^{-10}$. The set of parameters given in Table 1 were used in the simulations.

The linear tracking control is demonstrated using a reference signal that starts at zero, ramps up, oscillates at a specified frequency and then ramps down to zero. The control is turned on immediately at $t_{o}=0$. In Fig. 2 the reference signal is limited to small displacements (maximum displacement was $0.1 \mu \mathrm{m}$ ) so that the constitutive behavior is linear. In this case, excellent tracking is achieved as expected.

When the reference signal is increased to a level that induces hysteretic magnetic field-magnetization behavior, degradation in control authority occurs. Fig. 3 illustrates large error between
Table 1. PARAMETERS USED IN THE TRACKING CONTROL SYSTEM.

\begin{tabular}{l|l}
\hline Magnetostrictive and Damped Oscillator Parameters \\
\hline$k_{L}=1.5 \times 10^{7} \mathrm{~N} / \mathrm{m}$ & $a_{1}=1.5 \times 10^{-4} \mathrm{~N} / \mathrm{A}^{2}$ \\
$c_{L}=1.5 \times 10^{3} \mathrm{Ns} / \mathrm{m}$ & $c_{1}=6.1 \times 10^{-5} \mathrm{~m} / \mathrm{A}$ \\
$M_{L}=0.47 \mathrm{~kg}$ & $\bar{H}_{c}=3.3 \times 10^{3} \mathrm{~A} / \mathrm{m}$ \\
$M_{0}=2.75 \times 10^{5} \mathrm{~A} / \mathrm{m}$ & $c=0.65$ \\
$Y^{M}=3.0 \times 10^{10} \mathrm{~N} / \mathrm{m}^{2}$ & $b=2.25 \times 10^{4} \mathrm{~A} / \mathrm{m}$ \\
$A=5.0 \times 10^{-4} \mathrm{~m}^{2}$ & $L=0.1 \mathrm{~m}$ \\
$c_{D}=3.7 \times 10^{6} \mathrm{Ns} / \mathrm{m}$ & $\chi_{m}=15$ \\
\hline
\end{tabular}

the reference signal and the controlled displacement. In this simulation, the reference signal is specified to have a displacement amplitude of $100 \mu \mathrm{m}$. The hysteretic $H-M$ behavior is illustrated in Fig. 4.

\section{Nonlinear Optimal Tracking Control}

Nonlinearities and hysteresis are addressed in this section by implementing the constitutive behavior directly into the optimality system previously given by Eqs. (10) and (14). The methodology follows a previous approach for active vibration attenuation of beams and plates $[16,17]$. Key equations illustrating modifications needed to track a reference signal are presented.

In the nonlinear model, the force determined from Eq. (7) is included in the input operator $[B(u)](t)$ by linearizing about the bias field $M_{0}$ according to Eq. (17). This neglects the quadratic

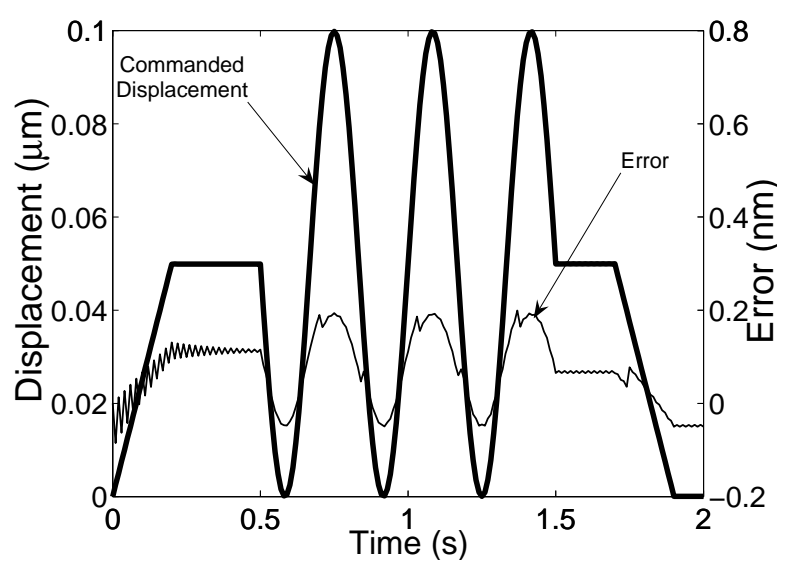

Figure 2. TRACKING CONTROL RESPONSE WHEN THE MAGNETOSTRICTIVE MATERIAL BEHAVIOR IS LIMITED TO THE LINEAR REGIME. THE CONTROLLED RESPONSE (IN $\mu \mathrm{m})(-$ AND THE ERROR BETWEEN THE REFERENCE SIGNAL AND CONTROLLED RESPONSE (IN nm) (-) IS SHOWN. 
coupling, but includes nonlinear and hysteretic field-coupled behavior. Details regarding this approximation are given in [18] and here in the Nonlinear Simulation Results. This yields the optimality system

$$
\begin{aligned}
& {\left[\begin{array}{l}
\dot{x}(t) \\
\dot{\lambda}(t)
\end{array}\right]=\left[\begin{array}{l}
A x(t)+[B(u)](t)+G(t) \\
-A^{T} \lambda(t)-C^{T} Q C x(t)+C^{T} Q r(t)
\end{array}\right]} \\
& x\left(t_{0}\right)=x_{0} \\
& \lambda\left(t_{f}\right)=C^{T} P\left(C x\left(t_{f}\right)-r\left(t_{f}\right)\right) .
\end{aligned}
$$

An efficient formulation in terms of a Ricatti equation is not possible in this case due to the nonlinear nature of the input operator. The two-point boundary value problem is addressed by approximating Eq. (26) or the equivalent first-order system,

$$
\begin{aligned}
& \dot{z}(t)=F(t, z) \\
& E_{0} z\left(t_{0}\right)=\left[x_{0}, 0\right]^{T} \\
& E_{f} z\left(t_{f}\right)=[0,0]^{T}
\end{aligned}
$$

where $z=[x(t), \lambda(t)]^{T}$.

The nonlinear optimality system is now given by

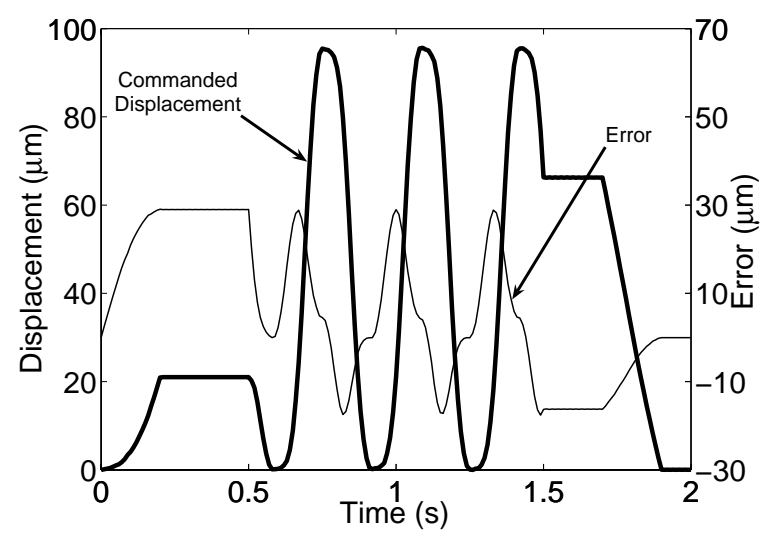

Figure 3. LINEAR TRACKING CONTROL RESPONSE WHEN MAGNETOSTRICTIVE NONLINEARITIES ARE PRESENT. THE CONTROLLED RESPONSE (IN $\mu \mathrm{m})(-)$ AND THE ERROR BETWEEN THE REFERENCE SIGNAL AND CONTROLLED RESPONSE (IN $\mu m)$ (-) IS SHOWN.

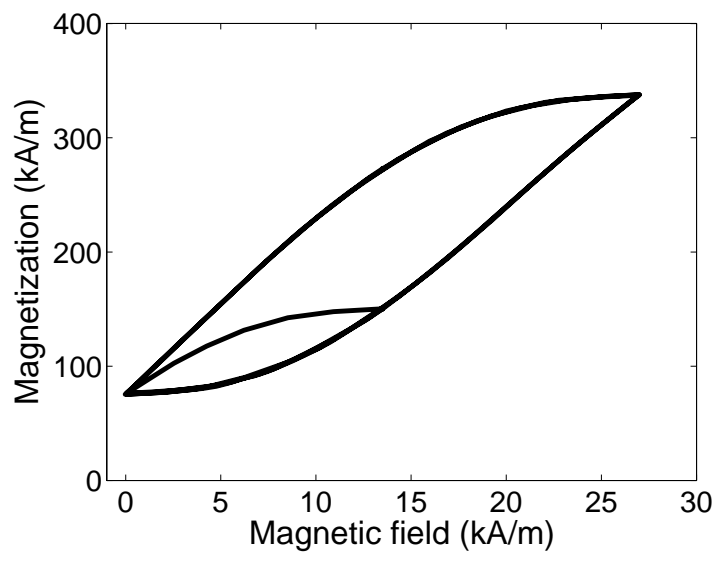

Figure 4. CONSTITUTIVE BEHAVIOR OF THE MAGNETOSTRICTIVE TRANSDUCER USED IN THE TRACKING CONTROL SIMULATION IN FIG. 3.

$$
\begin{aligned}
& F(t, z)=\left[\begin{array}{l}
A x(t)+[B(u)](t)+G(t) \\
-A^{T} \lambda(t)-C^{T} Q C x(t)+C^{T} Q r(t)
\end{array}\right] \\
& E_{0}=\left[\begin{array}{ll}
I & 0 \\
0 & 0
\end{array}\right], \quad E_{f}=\left[\begin{array}{rr}
0 & 0 \\
-C^{T} P C & I
\end{array}\right] .
\end{aligned}
$$

Here $I$ denotes an identity matrix with dimension corresponding to the number of basis functions employed in the spatial approximation of the state variables.

Various methods are available to approximate solutions to the system given by Eq. (27) such as finite differences and nonlinear multiple shooting [19]. The finite difference approach is used here where we consider a discretization of the time interval $\left[t_{0}, t_{f}\right]$ with a uniform mesh having stepsize $\Delta t$ and points $t_{0}, t_{1}, \cdots, t_{N}=t_{f}$. The approximate values of $z$ at these times are denoted by $z_{0}, \cdots, z_{N}$. A central difference approximation of the temporal derivative then yields the system

$$
\begin{aligned}
& \frac{1}{\Delta t}\left[z_{j+1}-z_{j}\right]=\frac{1}{2}\left[F\left(t_{j}, z_{j}\right)+F\left(t_{j+1}, z_{j+1}\right)\right] \\
& E_{0} z_{0}=\left[y_{0}, 0\right]^{T} \\
& E_{f} z_{N}=[0,0]
\end{aligned}
$$

for $j=0, \cdots, N-1$. 
Equation (29) can be expressed as the problem of finding $z_{h}=\left[z_{0}, \cdots, z_{N}\right]$ to which solves

$$
\mathcal{F}\left(z_{h}\right)=0
$$

Equation (30) includes the optimality system at each time step and the boundary conditions by manipulating Eq. (29). Details are given in [16].

A quasi-Newton iteration of the form

$$
z_{h}^{k+1}=z_{h}^{k}+\xi_{h}^{k}
$$

where $\xi_{h}^{k}$ solves

$$
\mathcal{F}^{\prime}\left(z_{h}^{k}\right) \xi_{h}^{k}=-\mathcal{F}\left(z_{h}^{k}\right)
$$

is then used to approximate the solution to the nonlinear system given by Eq. (30). The Jacobian $\mathcal{F}^{\prime}\left(z_{h}^{k}\right)$ has the form

$$
\mathcal{F}^{\prime}\left(z_{h}\right)=\left[\begin{array}{ccccc}
S_{0} & R_{0} & & & \\
& S_{1} & R_{1} & & \\
& & \ddots & \ddots & \\
& & & S_{N-1} & R_{N-1} \\
& & & & E_{f}
\end{array}\right]
$$

where

$$
S_{i}=-\frac{1}{\Delta t}\left[\begin{array}{ll}
I & 0 \\
0 & I
\end{array}\right]-\frac{1}{2}\left[\begin{array}{cc}
A & \frac{\partial}{\partial \lambda} B\left[u_{i}^{*}\right] \\
-C^{T} Q C & -A^{T}
\end{array}\right]
$$

for $S_{i}$. The representation for $R_{i}$ is similar.

Inversion of the Jacobian is required to obtain updates to the states through the iteration procedure. This is often not feasible when a large number of basis functions are required to discretized the structural model. In the one-dimensional rod model, this is typically not an issue, although to reduce potential numerical errors in inverting the Jacobian an analytic LU decomposition is employed. The form of the equation is identical to that used in a previous investigation [16]. This gives rise to the following representation of the Jacobian,

$$
\mathcal{F}^{\prime}\left(z_{h}^{k}\right)=L U
$$

The solution of the system (32) is then obtained through direct solution of the lower triangular system $L \zeta_{h}^{k}=-\mathcal{F}\left(z_{h}^{k}\right)$ followed by direct solution of the upper triangular system $U \xi_{h}^{k}=\zeta_{h}^{k}$.

Nonlinear Simulation Results The nonlinear optimal tracking control provides enhanced control when nonlinearities and hysteresis are present. The values for $Q$ and $R$ previously used in the linear model are used here in the nonlinear model. The performance enhancement using the nonlinear control design is demonstrated by prescribing the same reference signal that induced hysteresis in Fig. 4. The actuator nonlinearities are effectively compensated using the nonlinear optimal control method as shown in Fig. 5.

The tracking errors illustrated in Fig. 5 have been limited to less than $1.0 \mu \mathrm{m}$. These errors are primarily associated with unmodeled constitutive nonlinearities. As previously noted in the Nonlinear Optimal Track Control section, the control input is computed by approximating the strain to be proportional to the magnetization. This approximation is evaluated in Fig. 5 by computing the commanded displacement using the quadratic coupling behavior from Eq. 17. This is the primary source of error in the open loop case.

Improvements in error reduction and robustness to operating uncertainties can be achieved by feedback of perturbations in the optimal state trajectory. This has been recently addressed using a similar nonlinear optimal control design for vibration attenuation [17]. A hybrid method was adopted that computes the open loop nonlinear control off-line and perturbation feedback around

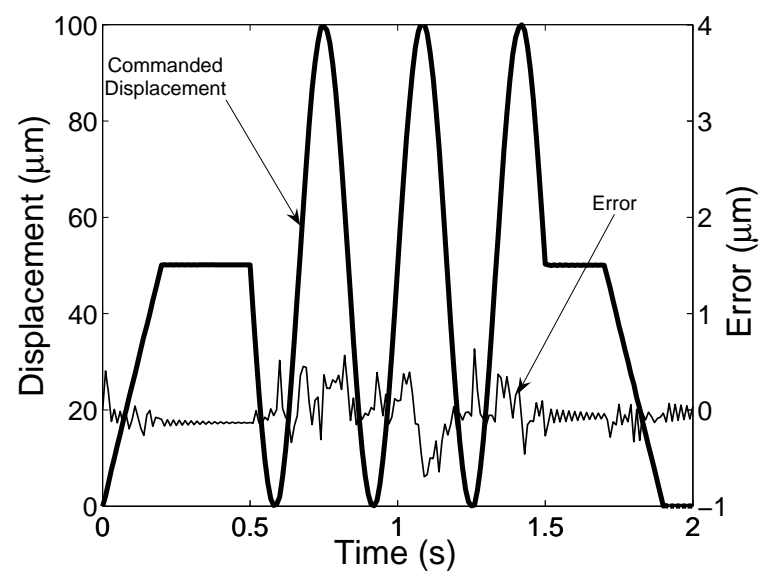

Figure 5. TRACKING CONTROL RESPONSE USING NONLINEAR CONTROL THEORY. THE CONTROLLED RESPONSE (IN $\mu \mathrm{m})(-)$ AND THE ERROR BETWEEN THE REFERENCE SIGNAL AND CONTROLLED RESPONSE (IN $\mu m)$ (-) IS SHOWN. 


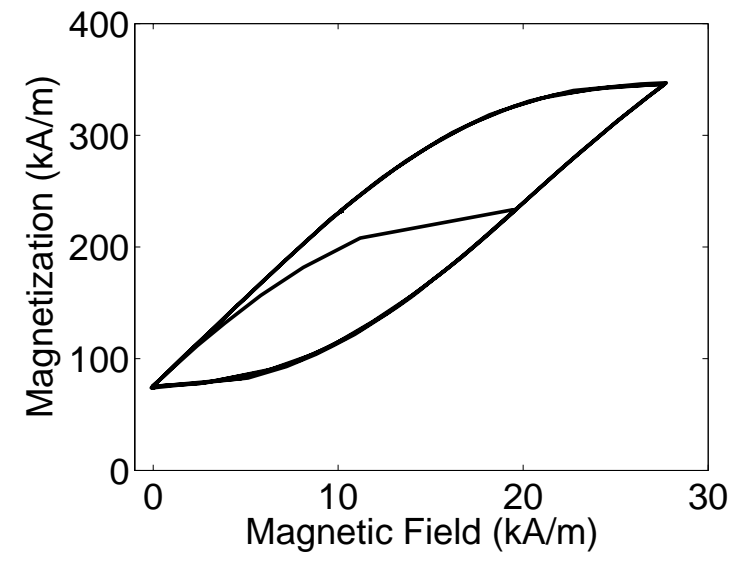

Figure 6. MAGNETIC FIELD-MAGNETIZATION CONSTITUTIVE BEHAVIOR CORRESPONDING TO THE TRACKING CONTROL SIMULATION IN FIG. 5.

the optimal trajectory. While there are similarities to the tracking problem, fundamental differences in the auxiliary equation (Eq. (25)) must be addressed. This is under current investigation.

\section{Concluding Remarks}

A nonlinear optimal tracking control design was developed to accurately track a reference signal when nonlinear, hysteretic magnetostrictive material behavior is present. It was shown that significant errors occur at moderate to high field inputs if the constitutive behavior is not compensated properly. This was addressed by determining the nonlinear input field from the homogenized energy model to compensate for nonlinear, hysteretic magnetostrictive constitutive behavior.

\section{ACKNOWLEDGMENT}

The authors gratefully acknowledge support through the Air Force Office of Scientific Research through grant AFOSRFA9550-04-1-0203.

\section{REFERENCES}

[1] Straub, F., Kennedy, D., Domzalski, D., Hassan, A., Ngo, H., Anand, V., and Birchette, T., 2004. "Smart materialactuated rotor technology-SMART". J. Intell. Mater. Syst. Struct., 15, pp. 249-260.

[2] Smith, R., Bouton, C., and Zrostlik, R., 2000. "Partial and full inverse compensation for hysteresis in smart material systems". Proc. 2000 American Control Conference, pp. 2750-2754.
[3] Smith, R., and Zrostlik, R., 1999. "Inverse compensation for ferromagnetic hysteresis". Proc. 38th IEEE Conference on Decision and Control, pp. 2875-2880.

[4] Duerig, T., 2002. "The use of superelasticity in modern medicine”. MRS Bull., 27(5), pp. 101-104.

[5] Ge, P., and Jouaneh, M., 1996. "Tracking control of a piezoceramic actuator". IEEE T. Contr. Syst. T., 4(3), pp. 209-216.

[6] Grant, D., and Hayward, V., 1997. "Variable structure control of shape memory alloy actuators". IEEE Contr. Syst. Mag., 17(3), pp. 80-88.

[7] Smith, R., 2001. "Inverse compensation for hysteresis in magnetostrictive transducers". Math. Comput. Model., 33, pp. 285-298.

[8] Tao, G., and Kokotović, P., 1996. Adaptive control of systems with actuator and sensor nonlinearities. John Wiley and Sons, New Jersey.

[9] Jiles, D., and Atherton, D., 1984. "Theory of the magnetomechanical effect”. J. Phys. D. Appl. Phys., 17, pp. 12651281.

[10] Smith, R., 2005. Smart Material Systems: Model Development. SIAM, Philadelphia, PA.

[11] Dapino, M., Smith, R., and Flatau, A., 2000. "A structural strain model for magnetostrictive transducers". IEEE T. Magn., 36(3), pp. 545-556.

[12] Bryson, A., and Ho, Y.-C., 1969. Applied Optimal Control. Blasidell Publishing Company, Waltham, MA.

[13] Lewis, F., and Syrmos, V., 1995. Optimal Control. John Wiley and Sons, New York, NY.

[14] Bittanti, S., Locatelli, A., and Maffezzoni, C., 1972. "Periodic opimization under small perturbations". In Periodic Optimization, A. Marzollo, ed., Vol. II. Udine, SpringerVerlag, New York, pp. 183-231.

[15] DaPrato, G., 1987. "Synthesis of optimal control for an infinite dimensional periodic problem". SIAM J. Control Optim., 25(3), pp. 706-714.

[16] Smith, R., 1995. "A nonlinear optimal control method for magnetostrictive actuators". J. Intell. Mater. Syst. Struct., 9(6), pp. 468-486.

[17] Oates, W., and Smith, R. "Nonlinear optimal control techniques for vibration attenuation using nonlinear magnetostricive actuators". submitted to J. Intell. Mater. Sys. Struct.

[18] Nealis, J., and Smith, R. "Model-based robust control design for magnetostrictive transducers operating in hysteretic and nonlinear regimes". submitted to IEEE Trans. Control Syst. Technol.

[19] Ascher, U., Mattheij, R., and Russell, R., 1995. Numerical Solution of Boundary Value Problems for Ordinary Differential Equations. SIAM Classics in Applied Mathematics. 